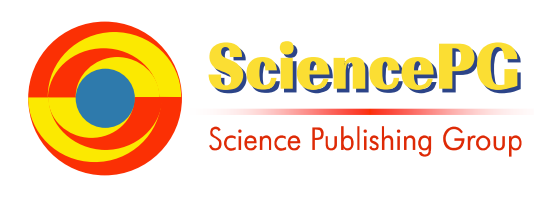

\title{
Matrix Shear-Lag Parameter in a Shape Memory Alloy-Actuator-Reinforced Silicon Elastomer
}

\author{
Michael. O. Obaji \\ Department of Precision Machinery and Instrumentation, University of Science and Technology of China, Hefei, China \\ Email address: \\ obajimo@yahoo.com

\section{To cite this article:} \\ Michael. O. Obaji. Matrix Shear-Lag Parameter in a Shape Memory Alloy-Actuator-Reinforced Silicon Elastomer. International Journal of \\ Materials Science and Applications. Vol. 5, No. 5, 2016, pp. 194-201. doi: 10.11648/j.ijmsa.20160505.13
}

Received: August 11, 2016; Accepted: August 22, 2016; Published: September 9, 2016

\begin{abstract}
The excellent qualities possessed by silicon elastomer when used in designing flexible parts of mechanical systems, make it imperative that we analyze its deformations and distributed axial forces in a matrix of shape memory alloy (SMA) fibers designed as possible appendages for gripping robots. The essence of these analyses is to determine the shear-lag parameter which has influence on the axial distributed forces proposed as a gauge for testing the structure in a high yield, high force and high strain mechanical environment. The insertion of SMA fibers in flexible rods cast using silicon elastomer results in the deformation of the host medium once shape recovery of the fiber occurs. This paper aims to analyze the mechanics of the said shape recovery in a modeled silicon elastomer rod with a single off-axis reinforced SMA actuator. The compressive force distribution mechanism and the bending moment caused by phase transformation in the design are determined using an approximate analytical model. The deformations on the structure proposed as an appendage on gripping robots were further analyzed by determining their equations of equilibrium, force factors and their comparative shear-lag models to be able to estimate the force distribution on the structure.
\end{abstract}

Keywords: SMA Actuator, Mechanics, Silicon Elastomer, Deformation, Soft Robots

\section{Introduction}

The phase changes often associated with SMA fibers via heating and cooling cycles is usually as a result of electrical heating from martensite to austenite. This is what causes the bending of the SMA fibers [8]. And because resistive heating generated by an electric current is the major driving force for their actuation, it thus becomes somewhat easier to drive the fibers, especially when embedded in a rod [5]. SMA fibers are known to exert high force and allow for high strain, thus making their insertion in silicon elastomer rods an interesting area of research, especially in designing mechanical systems.

Silicone elastomer is a polymeric material with visco-elasticity made of poly-siloxanes obtained from ketones and monomers such as carbon, hydrogen and oxygen. They have low Young's modulus and high yield strain compared with any other materials [9]. When cast at ambient temperatures, silicon elastomers are relatively soft and deformable. They are mainly used for molding flexible parts in aircrafts, seals in building construction \& maintenance and adhesives in cars. They are also widely used in producing medical grade materials for treating patients in hospitals. Silicon elastomers are thermosets by nature, which means they are vulcanizable but could also be thermoplastic. Their molecular structure look like the spaghetti and meatball cooked as food in our homes. The meatball serves as a cross-link (or chain) which gives it the elastic property. This elastic property comes from the ability of the long chains to reconfigure themselves and distribute applied stress [Keller et al]. The covalent chains (or cross-linkages) ensure that the elastomer returns to its original configuration when the stress is removed. Thus, making its shape-recovery features similar to that of the SMA fiber. Although, the insertion of SMA fibers in silicon elastomer for the purpose of developing appendages for mechanical systems such as robots is still a novel idea being researched on, there is need to emphasize that some breakthroughs similar to this had been made in designing appendages for robots, although not with silicon elastomer, as Hitachi produced a 4-fingered robotic hand using 12 groups of $0.2 \mathrm{~mm}$ SMA fibers that closed their hands upon activation. Similarly, there was an SMA-Based 
Six-Legged Biomimetic Robot called SMABOT built by the National Taiwan University. There was also a 4-Degree of Freedom Origami-enabled, SMA-actuated Robotic End-effectors for Minimally Invasive Surgery designed in King's College London. All these feats so far achieved encourage more researchers to exploit the possibility of using elastomeric materials reinforced with SMA actuators in building components for mechanical systems such as robots. An accurate analysis of the force distribution and shear-lag parameter on the modeled structure which could help determine its effectiveness when designing mechanical systems is a case for concern, and this has to be investigated. This paper will also investigate the nagging issue of how to determine SMA fiber placement in a rod of a given shape.

\section{Design and Deformation}

The embedding of SMA fiber in a flexible rod is complex due to force transfer mechanism from the actuator to the rod $[2,11]$. However, in order to deal with this complexity, we have to consider a single SMA fiber embedded in a flexible rod as shown in figure 1(a).

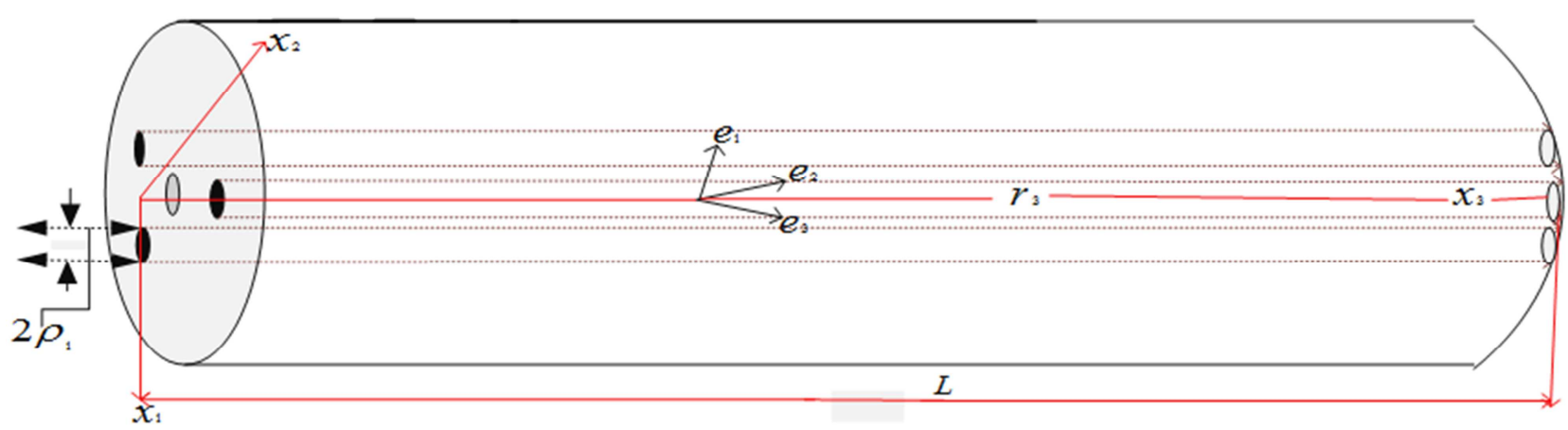

(a)

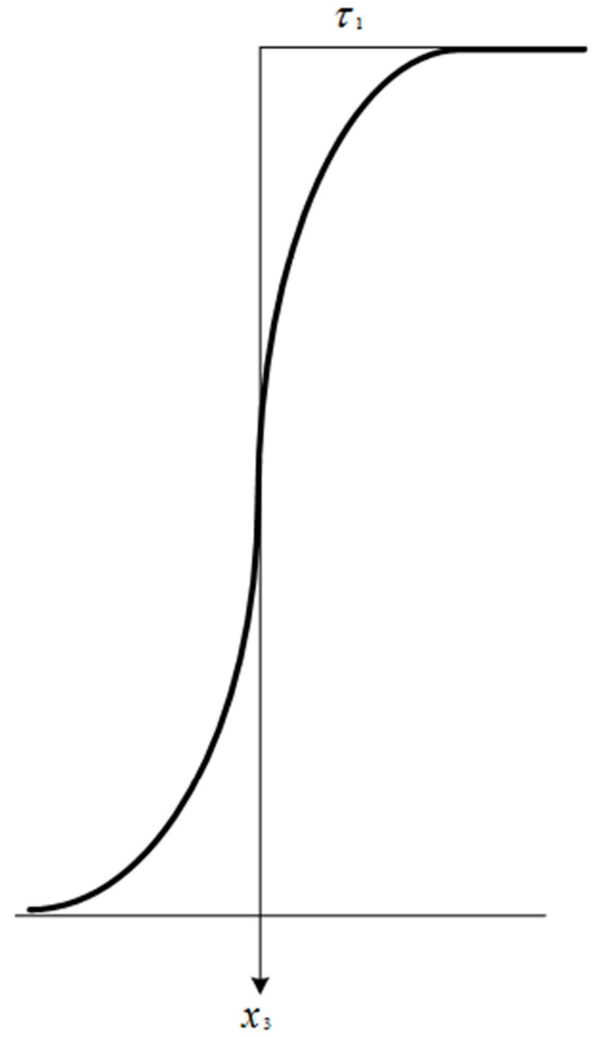

(b)

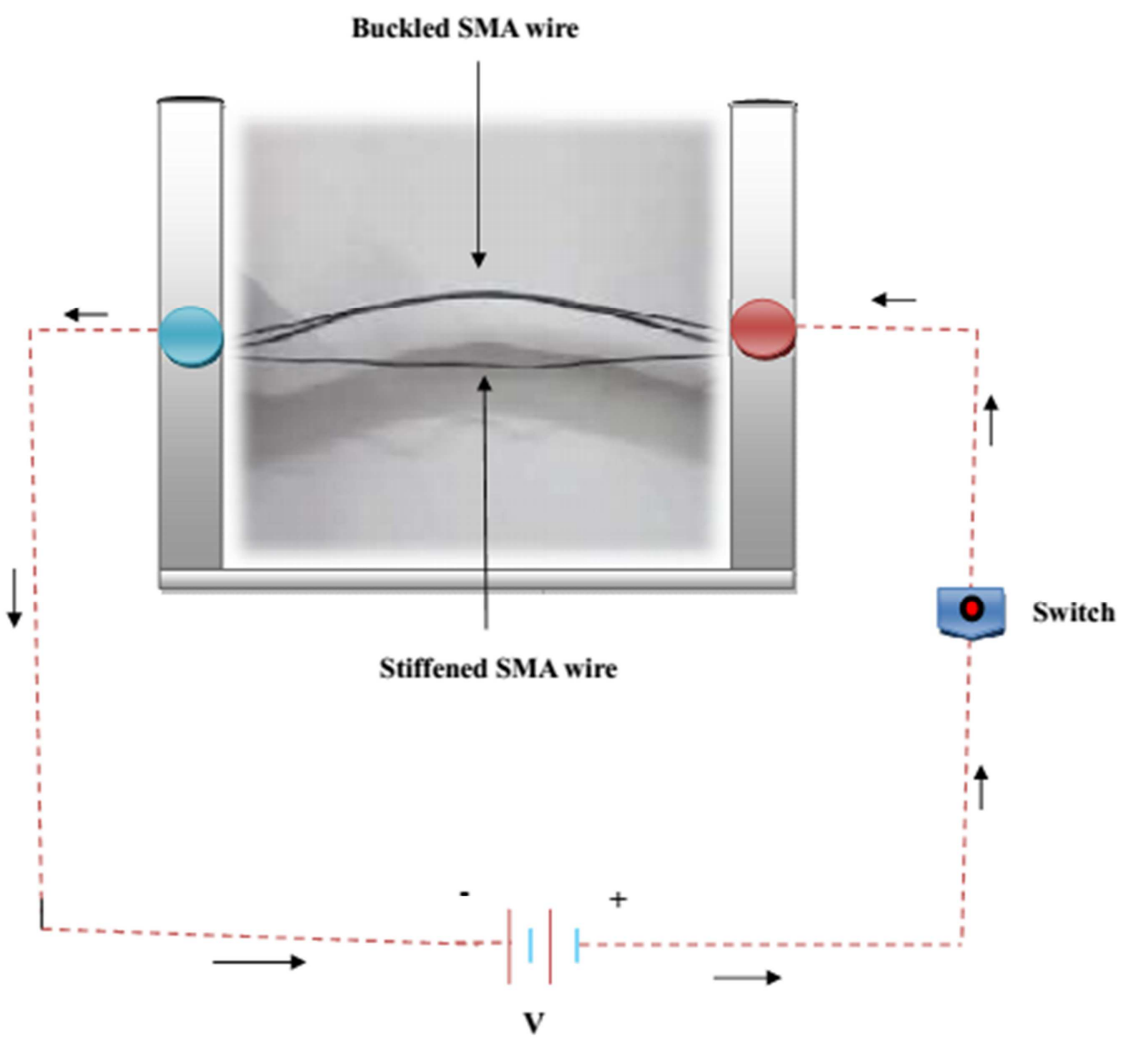

(c)

Figure 1. (a) Shows the fiber and rod coordinate system with the off-axis actuating fiber inserted. (b) Shows the change in shape of a flexible rod when inserted with an actuating fiber. (c) A circuit showing SMA fiber deformation upon receiving current.

Further to this, a suitable model for the force transfer from the SMA fiber to the cylindrical host medium is the cylindrical shear-lag model [1]. According to this model, the axial strain of the cylinder and the partial shape recovery of SMA occur by the deformation of a shear layer around the SMA fiber.

In this paper, we shall establish the equilibrium equations for the shear layer, the host medium, and the SMA fiber. 


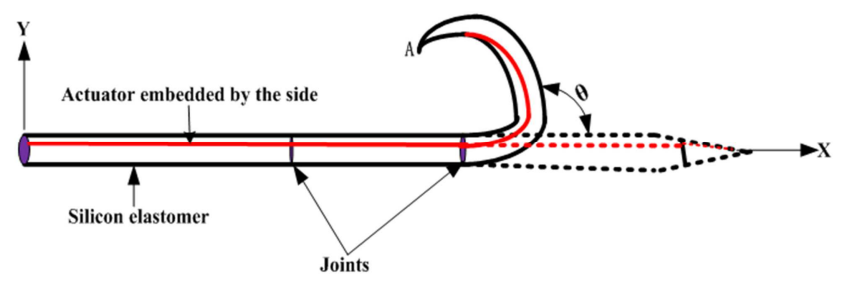

Figure 2. The deformation of a silicon elastomer rod.

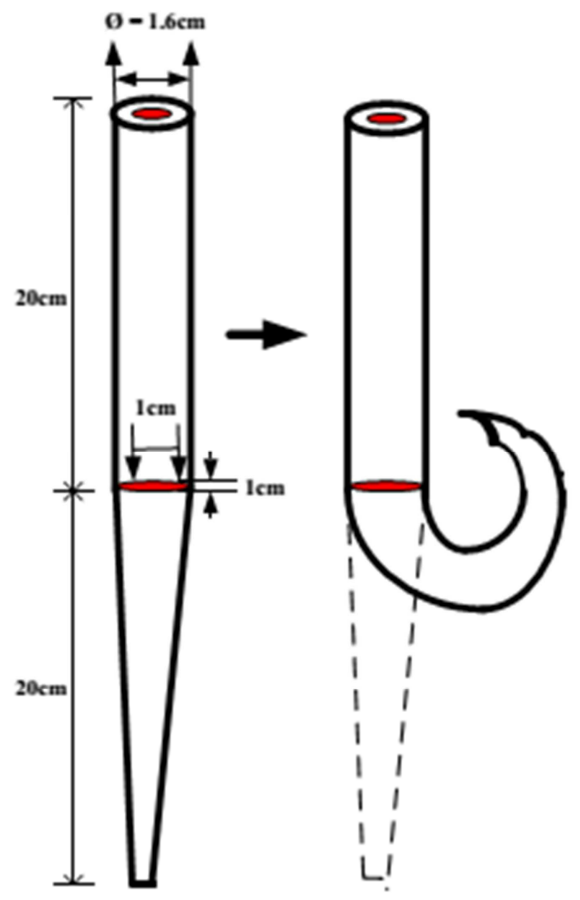

Figure 3. Schematic showing the dimensions of the model.

The cylinder cast using silicon elastomer is intended to imitate the appendage of an octopus or that of an octopus robot.

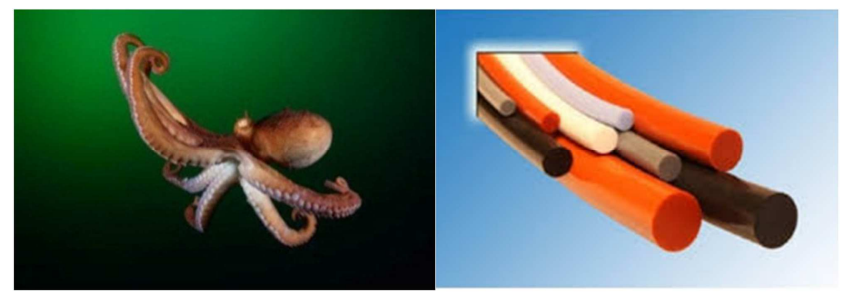

Figure 4. (a) An octopus showing arm-deformation caused by internal compressive loading. (b) Typical silicon elastomer rods.

\section{Mechanism and Mathematical Analysis}

During force transfer from SMA fiber to the silicon elastomer rod, there is usually an existence of initial permanent strain which normally occurs when they are well-bonded to the rod during martensite phase [1]. Under this condition, we also assume that there exists a stress-free initial combined structure. Thus, if the temperature is raised above austenitic finish for the combined structure, there will be an inducement of strain around the host medium, as a result of changes in length of the fiber connected with the martensite-austenite phase changes. Further to this, we assume that the fiber is in the martensite state, with an initial permanent axial strain represented by $-\mathcal{E}^{S M A}$. This symbol also represents the strain associated with the stress-free shape recovery when the heating temperature rises above the austenitic finish. The strain would be recovered as long as the SMA fiber could undergo the martensitic-austenitic phase changes.

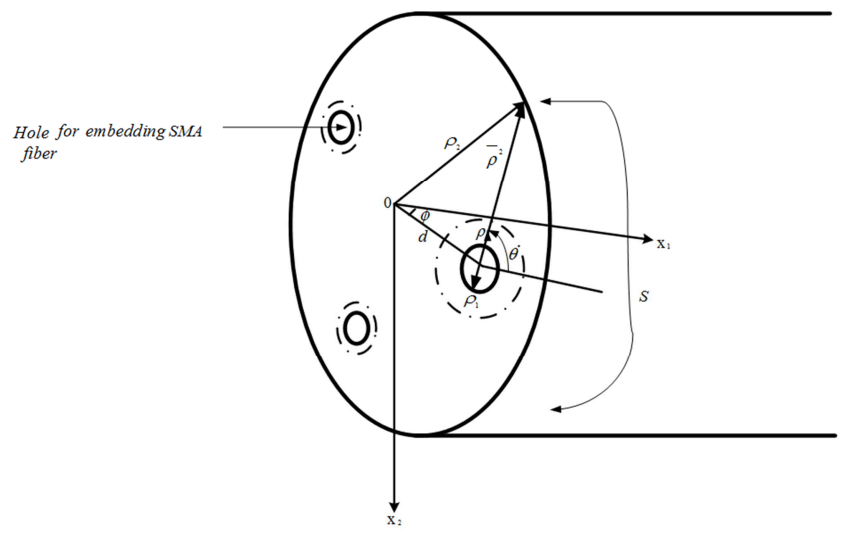

Figure 5. Force transfer from actuating fiber to the flexible rod showing their shear-lag forms.

As a result of the constraint introduced by the elastomeric rod hosting the actuator, a self-imposed equilibrium state of internal stress will instead develop. And the pre-strain $\mathcal{E}^{S M A}$ will be recovered partially.

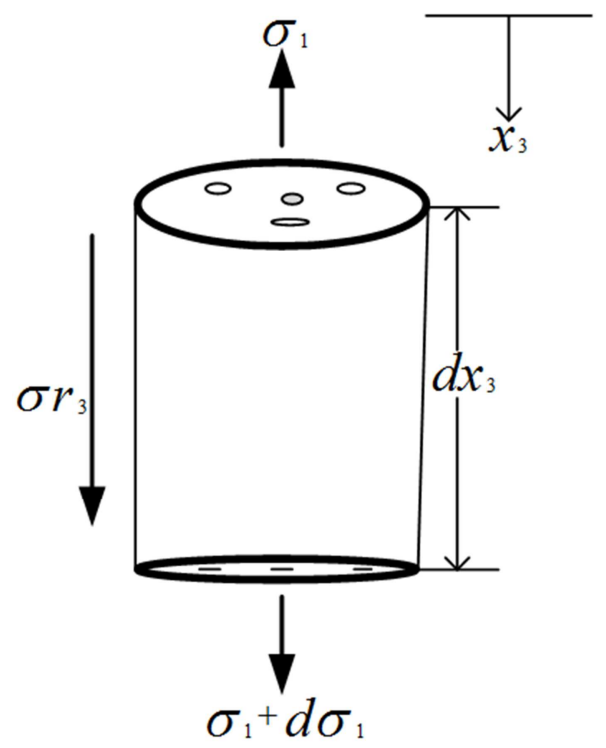

Figure 6. Force transfer from actuating fiber to the rod with their geometric parameters.

Thus, the equations of equilibrium state of internal stress created by the cylindrical host medium, the shear layer and the SMA fiber are related as follows: 


$$
\begin{gathered}
\frac{d \sigma_{1}}{d x_{3}}+\left.\frac{2}{\rho_{1}} \sigma r_{3}\right|_{r=\rho_{1}}=0 \quad 0 \leq x_{3} \leq \mathbf{L} \\
\frac{d \sigma r_{3}}{d r}+\frac{1}{r} \sigma r_{3}=0 \quad \rho_{1} \leq r \leq \rho(\theta) \\
\frac{d \sigma_{2}}{d x_{3}}-\left.\frac{2 \rho}{\bar{\rho}_{2}^{2}-\rho_{1}^{2}} \sigma r_{3}\right|_{r=\rho}=0 \quad 0 \leq x_{3} \leq \mathbf{L}
\end{gathered}
$$

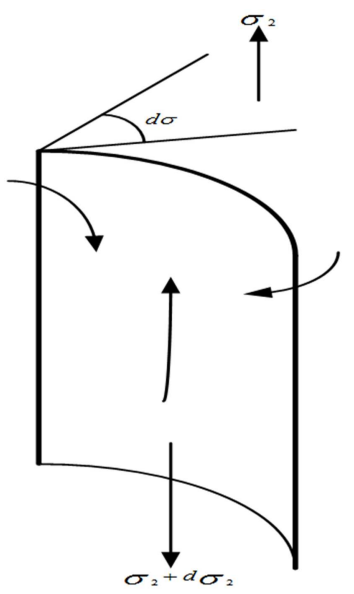

Figure 7. Structural changes due to shear caused by force transfer.

The equilibrium force in the axial direction of rod and the angular change, $d \theta$ of the SMA fiber have the following relationship:

$$
\rho_{1}^{2} \sigma_{1}+\left(\bar{\rho}_{2}^{2}-\rho_{1}^{2}\right) \sigma_{2}=0
$$

Where $\rho_{1}$ is the radius of the fiber; $\rho_{2}$ is the rod radius; $\overline{\rho_{2}}(\theta)$ is deduced from $\sqrt{\left(\rho_{2}^{2}-d^{2} \sin ^{2} \theta\right)}-d \cos \theta$ (refer to figures $5,6$ and 7$)$.

Furthermore, $d$ represents the distance between SMA fiber axis and the central axis of the rod, as seen in figure 5.

$\rho(\theta)$ is the shear-lag parameter and perhaps, a function of $\theta ; \sigma_{1}$ is the normal stress in the actuator in the axial direction and $\sigma_{2}$ is the normal stress in the axial direction in the fiber; $\sigma_{r 3}$ represents the shear stress in the shear layer which transfers the force from the actuating fiber to the cylindrical rod.

The actuating fiber and the cylindrical rod are related via a constitutive equation given as follows:

$$
\begin{gathered}
\sigma_{1}=E_{1} \frac{d w_{1}}{d z}-E_{1} \varepsilon^{S M A} \\
\sigma_{2}=E_{2} \frac{d w_{2}}{d z} \\
\sigma r_{3}=G_{2} \frac{\partial w}{\partial r}
\end{gathered}
$$

where $E_{1}$ is the Young's modulus of the actuating fiber in the austenite phase; $E_{2}$ is the Young's modulus of the elastomeric $\operatorname{rod}$ and $G_{2}$ is the shear modulus of the rod.

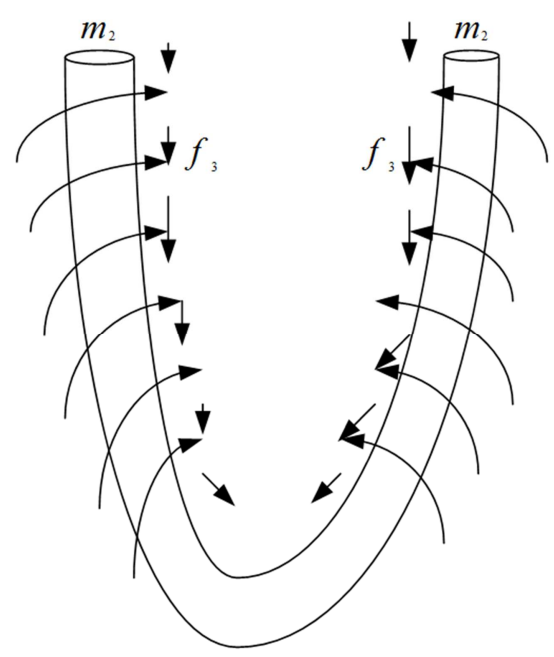

Figure 8. Diagram showing deformation and bending moment in the rod caused by SMA fiber actuation.

The fiber, the host medium and the shear layer have axial displacements represented as $\mathcal{W}_{1}\left(x_{3} ; \theta\right) ; \mathcal{W}_{2}\left(x_{3} ; \theta\right)$ and $w\left(x_{3}, r ; \theta\right)$, respectively. Since $\rho$ and $\overline{\rho_{2}}$ depend on $\theta$, the axial displacements will also depend on $\theta$.

The above equations have been solved by integration, using a general concept of an off-axis inserted SMA actuator. The concept is by first of all integrating equation (2), and getting its outcome embedded in (1), while (4) is applied, followed by the constitutive equations (5-7). Thus:

$$
\frac{d^{2} \sigma_{1}}{d x_{3}^{2}}-\alpha^{2} \sigma_{1}=-\alpha^{2} \sigma^{0}
$$

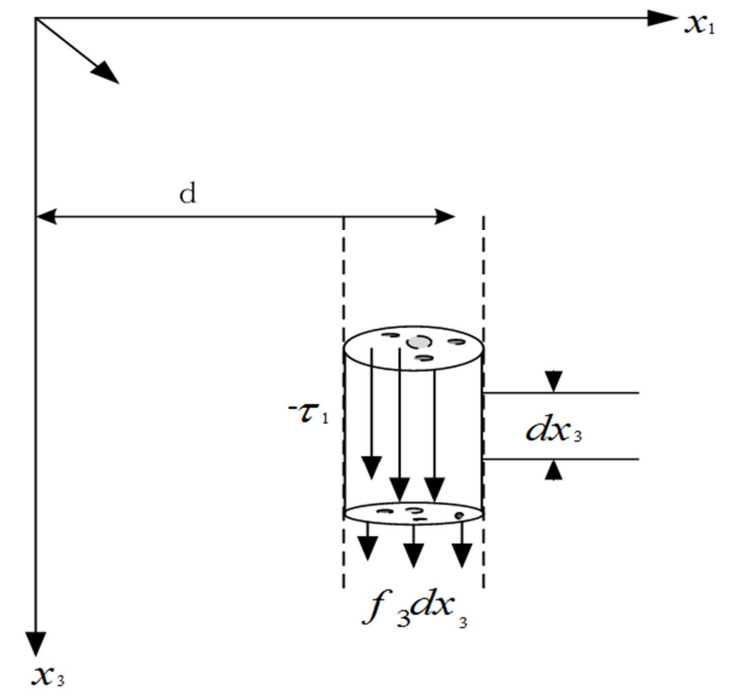

Figure 9. Schematic of axial compressive force showing their distributions.

where $1 / \alpha$ is a typical length of the problem described by

$$
\alpha^{2}=\frac{2 G_{2}}{E_{2} \rho_{1}^{2} \ln \left(\rho / \rho_{1}\right)}\left(\frac{E_{2}}{E_{1}}+\frac{\rho_{1}^{2}}{\bar{\rho}_{2}^{2}-\rho_{1}^{2}}\right)
$$


and $\sigma^{0}$ represents the stress in the actuating fiber away from the ends, summarized by:

$$
\sigma^{0}=-E_{2} \varepsilon^{\text {SMA }} /\left(\frac{E_{2}}{E_{1}}+\frac{\rho_{1}^{2}}{\bar{\rho}_{2}^{2}-\rho_{1}^{2}}\right)
$$

By applying the boundary conditions, $\sigma_{1}^{(0)}=0, \sigma_{1}(\mathrm{~L})=0$; then equation (8) is resolved into:

$$
\sigma_{1}^{(S)}=\sigma^{0}\left(1-\frac{e^{-\alpha} x_{3}-e^{-\alpha L+\alpha} x_{3}}{1+e^{-\alpha L}}\right)
$$

The shear-lag parameter $\rho$ in equation (11) remains the only undetermined value. But in an off-axis actuator inserted in a circular rod, $\rho$ is assumed to be equivalent to $\left(\rho_{2}-d\right)$.

The interface shear stress between the actuating fiber and the rod is determined from (1) when (11) is substituted thereat, and then:

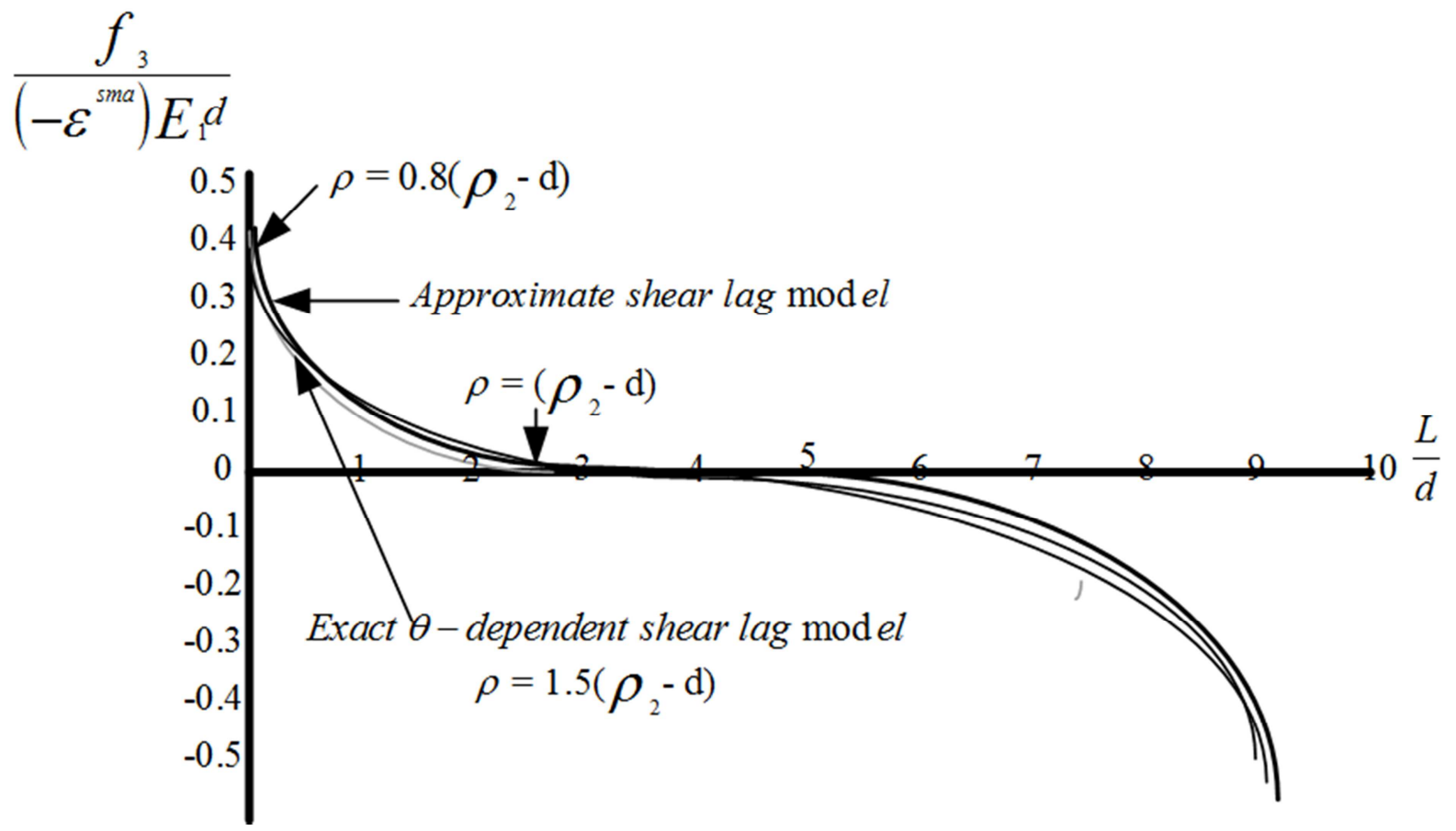

Figure 10. Shows how shear-lag parameter influences the axial distributed forces with comparison between the exact and the approximate.

Equation (13) above was numerically integrated via Mathematica (Wolfram 1991).

And because of the smallness of the difference between the exact and the approximate, it is justifiable to use the simpler approximate formula for $f_{3}$ as an estimate for force distribution on the structure. Figure 10 above, further explains the use of shear-lag parameter $\rho$, where $f_{3}$ generates a graph from the following cases: $\rho=\rho_{2}-\mathrm{d}, \rho=0.8\left(\rho_{2}-d\right)$ and $\rho=1.5\left(\rho_{2}-d\right)$. The sensitivity of $f_{3}$, which is small to $\rho$, is explained by the solution which depends on $\rho$ logarithmically.

With reference to the coordinate system chosen in figure 1 ,

$$
\left.\tau_{1\left(x_{3}\right)} \equiv \sigma r_{3}\right|_{r \equiv \rho_{1}}=-\sigma^{0} \frac{\rho_{1}}{2} \frac{\alpha e^{-\alpha x_{3}}-e^{-\alpha L+\alpha x_{3}}}{1+e^{-\alpha L}}
$$

Because of the interface stress created, a distributed bending moment and a distributed axial force is applied on the cylindrical rod by the SMA fiber (see figure 8).

The distributed axial force which was deduced from the integration of the interface shear upon the boundary of the actuating fiber through the axis of the rod becomes:

$$
f_{3}=-\int_{0}^{2 \pi} \tau_{1} \rho_{1} d \theta \cong \sigma^{0} \pi \rho_{1}^{2} \alpha \frac{e^{-\alpha} x_{3}-e^{-\alpha L+\alpha} x_{3}}{1+e^{-\alpha L}}
$$

The formula above, which is approximate for $f_{3}(x)$ is obtained, if $\rho_{2}$ replaces $\bar{\rho}_{2}^{(\theta)}$ in the solutions of $\alpha$ and $\sigma^{0}$.

Figure 10 below shows a comparison between the approximate formula for $f_{3}(x)$ and the exact. the distributed bending moments will have the following components (see figures 5, 6, 7, and 8):

$$
\begin{aligned}
& m_{1}=-\int_{0}^{2 \pi} \tau_{1} \rho_{1}\left(d \sin \varphi-\rho_{1} \sin \theta\right) d \theta \cong d \sin \varphi f_{3}\left(x_{3}\right) \\
& m_{2}=\int_{0}^{2 \pi} \tau_{1} \rho_{1}\left(d \cos \varphi+\rho_{1} \cos \theta\right) d \theta \cong-d \cos \varphi f_{3}\left(x_{3}\right)
\end{aligned}
$$

\section{Experiments}

SMA fiber was inserted off-axially in a cylindrical rod cast with silicon elastomer and modeled as an appendage for a gripping robot. It was driven electrically to test its bending 
moment and gripping capability. Their deformations were measured as seen in figures $11 \& 12$, with readings taken there from.

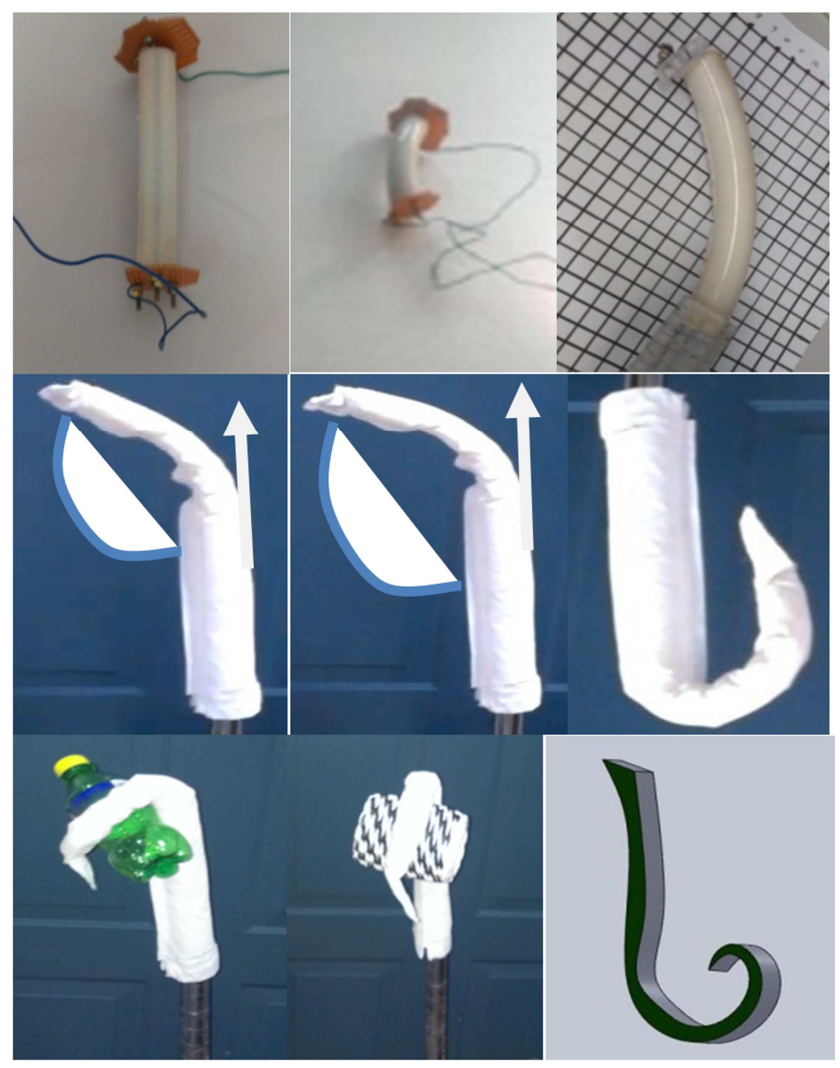

Figure 11. Experimental determination of the deformations of the SMA fiber-actuated rods and their gripping ability. Inset has a 3-D design of the modeled arm.

The equilibrium equations of a rigid body that has distributed bending moments and applied distributed forces and a quadratic strain energy function are expressed as follows.

$$
\begin{gathered}
F_{1}^{\prime}+K_{2} F_{3}-K_{3} F_{2}+f_{1}=0 \\
F_{2}^{\prime}+K_{3} F_{1}-K_{1} F_{3}+f_{2}=0 \\
F_{3}^{1}+K_{1} F_{2}-K_{2} F_{1}+f_{3}=0
\end{gathered}
$$

$$
\begin{gathered}
E_{2} I_{11} K_{1}^{\prime}+G_{2} I_{33} K_{2} K_{3}-E_{2} I_{22} K_{2} K_{3}-F_{2}{ }^{+} m_{1}=0 \\
E_{2} I_{22} K_{2}^{\prime} E_{2} I_{11} K_{1} K_{3}-G_{2} I_{33} K_{1} K_{3}{ }^{+} F_{1}{ }^{+} m_{2}=0 \\
G_{2} I_{33} K_{3}^{\prime}{ }^{+} E_{2} I_{22} K_{1} K_{2}-E_{2} I_{11} K_{1} K_{2}{ }^{+} m_{3}=0
\end{gathered}
$$

In the light of the above equations, $F_{1}(S)$ and $F_{2}(S)$ are the shear forces in the robot arm while $F_{3}(S)$ is the axial force in the arm [6].

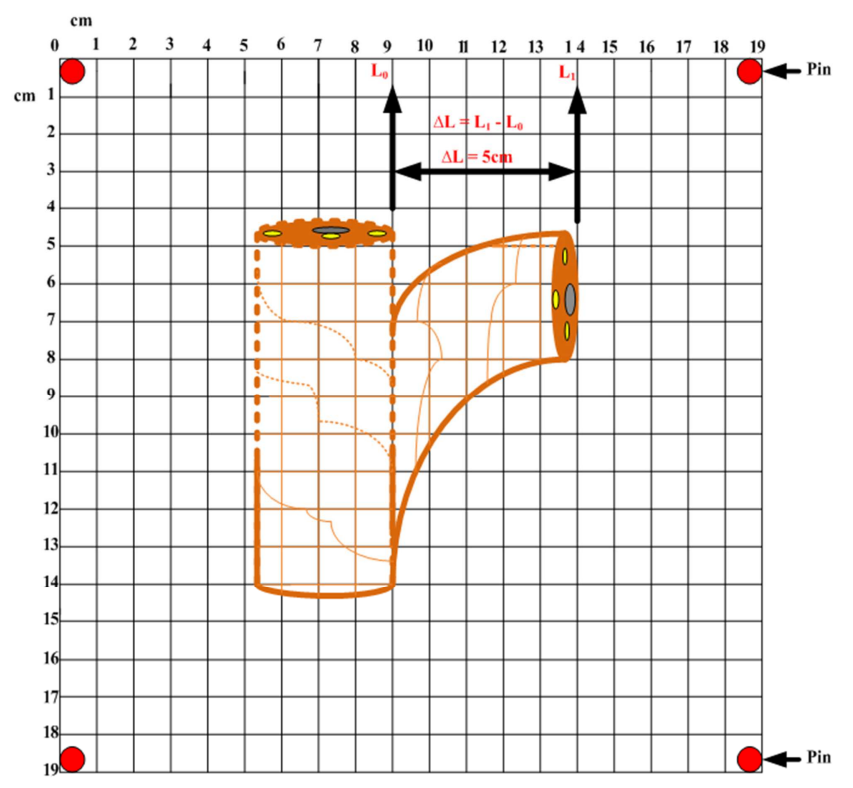

Figure 12. Schematic to measure the deformation of the SMA fiber-actuated rod.

$K_{1}{ }^{(S)}, K_{2}{ }^{(S)}$ and $K_{3}{ }^{(S)}$ are the curvatures and twist of the deformed shape of the structure, respectively (see figure 11).

$I_{11}, I_{22}$ and $I_{33}$ are the cross-sectional moments of inertia of the structure with respect to the principal axes, and the polar moment of inertia, respectively.

In the same vein, $f_{1}, f_{2}, f_{3}$ represent the external forces distributed on the structure while $m_{1}, m_{2}, m_{3}$ refer to couples on the structure.

Furthermore, $G_{2}$ and $E_{2}$ refer to the shear Modulus and Young's Modulus of the structure respectively.

Considering an off-axis SMA actuator inserted in a straight elastomeric rod, with a cylindrical configuration, the coordinate system can be chosen such that $f_{1}=f_{2}=0$, $m_{1}=m_{3}=0$, while $\mathrm{s}$ represents the arc length of the central axis connecting the the structure (see figure 5). Thus, equations (13) and (14ii) will beget $f_{3}(S)$ and $m_{2}(S)$ respectively, if $\mathrm{s}$ replaces $x_{3}$

The resultant equations of equilibrium of couple of forces on the structure, under this condition would resolve into the following:

$$
\begin{gathered}
F_{1}^{\prime}+K_{2} F_{3}=0 \\
F_{3}^{\prime}-K_{2} F_{1}+f_{3}=0 \\
E_{2} I_{22} K_{2}^{\prime}+F_{1}+m_{2}=0
\end{gathered}
$$

Equations (21), (22) \& (23) are non-linear Ordinary Differential

Equations. They are supported by the following kinematic equations:

$$
k_{2}=\varphi_{2}
$$




$$
\begin{gathered}
x_{1}=\int_{0}^{s} \sin \varphi_{2} d s \\
x_{3}=\int_{0}^{s} \cos \varphi_{2} d s
\end{gathered}
$$

where $\varphi_{2}$ is the cross sectional rotation of the structure about $x_{2}$ axis.

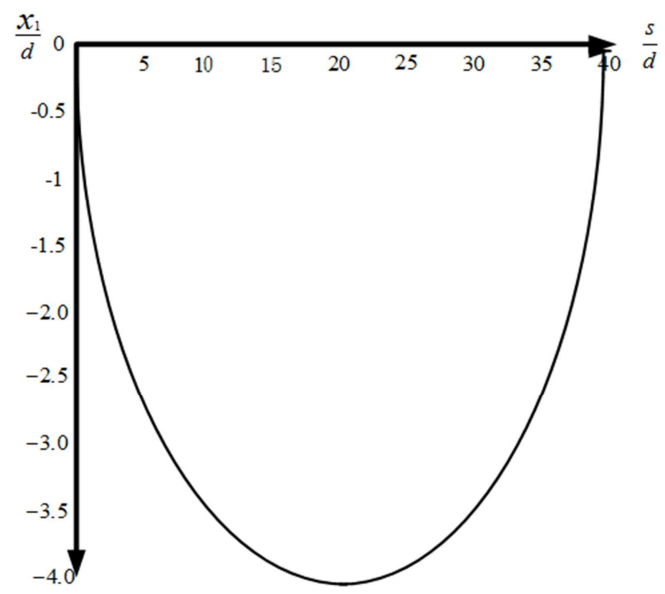

Figure 13. Deformed shape of the structure with an embedded off-axis SMA fiber inserted at one end.

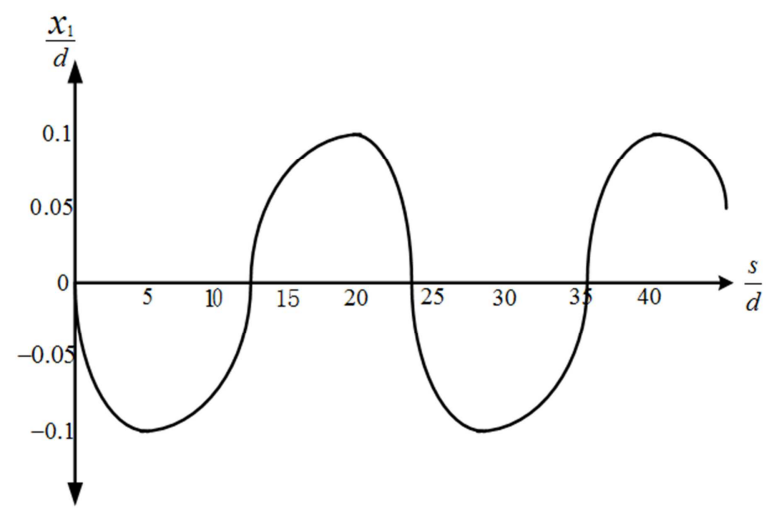

Figure 14. Deformed shape of the structure with an off-axis SMA actuator inserted at opposite ends.

\section{Conclusion}

An appendage to imitate the bending and gripping tendency of a soft robot such as octopus or elephant tusk was designed and modeled. Their deformations, bending moment and compressive force distribution mechanism were analyzed using an approximate analytical model to determine their comparative shear-lag parameter, with a view to determining their fitness in a mechanical environment where there is need for high yield, high force and high strain.

By considering a finite length of the structure designed, equations (21) - (26), which are Ordinary Differential Equations, were numerically integrated by applying a $4^{\text {th }}$ order Runge-Kutta scheme. [7].

All calculations were symbolically done via Mathematica
The integration of equations (21) - (26) was done from $\mathrm{S}=0$ to $\mathrm{S}=\mathrm{L}$ via an initial presumption for $F_{1}(0), F_{2}(0)$ and $F_{3}(0)$; but $x_{1}{ }^{(L)}, F_{3}(L)$ and $k_{2}(L)$ had an error which was used to fill-in $F_{1}{ }^{(0)}, F_{3}{ }^{(0)}$ and $\varphi_{2}{ }^{(0)}$ by adding some increments to them gotten from the solution to a system of linear equations shown as below:

$$
\left[\begin{array}{lll}
\frac{\partial x_{1}^{(L)}}{\partial F_{1}(0)} & \frac{\partial x_{1}^{(L)}}{\partial F_{3}(0)} & \frac{\partial x_{1}^{(L)}}{\partial \varphi_{2}{ }^{(0)}} \\
\frac{\partial F_{3}(L)}{\partial F_{1}(0)} & \frac{\partial F_{3}^{(L)}}{\partial F_{3}(0)} & \frac{\partial F_{3}(L)}{\partial \varphi_{2}(0)} \\
\frac{\partial k_{2}(L)}{\partial F_{1}(0)} & \frac{\partial k_{2}^{(L)}}{\partial F_{3}(0)} & \frac{\partial k_{2}(L)}{\partial \varphi_{2}(0)}
\end{array}\right]\left[\begin{array}{l}
\partial F_{1}(0) \\
\partial F_{3}(0) \\
\partial \varphi_{2}^{(0)}
\end{array}\right]=\left[\begin{array}{l}
-x_{1}{ }^{(L)} \\
-F_{3}{ }^{(L)} \\
-k_{2}(L)
\end{array}\right]
$$

\section{Acknowledgements}

*This work was supported by the National Natural Science Foundation of China (No. 50975270) and the Fundamental Research Funds for the Central Universities of China (No. WK2090090002).

\section{References}

[1] Budiansky, B., Hutchinson J. W. and Evans, A. G. (1986). "Matrix fracture in fiber-reinforced ceramics". J. Mech. Phys. Solids 34 167-89.

[2] K. Yang and C. L. Gu. "Design, drive and control of a novel SMA-actuated humanoid flexible gripper", J. Mech, Sci. Technol., vol. 22, no. 5, pp. 895-904, May 2008.

[3] Denny, M. W. 1988. "Biology and the Mechanics of the Wave-swept Environment"; Princeton University Press, Princeton, NJ.

[4] J. Jayender, R. V. Patel, S. Nikumb, and M. Ostojic, "Modeling and Control of Shape Memory Alloy Actuators", IEEE Trans. Control Syst. Technol., vol. 16, no. 2, pp. 279-287, Mar. 2008.

[5] Toi, Y., Lee, J. B, and Taya, M. (2004). "Finite element analysis of superelastic, large deformation behavior of shape memory alloy helical springs" Comput. Struct. 82 1685-93.

[6] Love, A. E. H. 1944. "A Treatise on the Mathematical Theory of Elasticity” (New York: Dover).

[7] Wolfram Research, Inc., 1991 Mathematica, Version 2.0 (Wolfram Research, Inc., Champaign, Illinois).

[8] Jackson, C. M., H. J. Wagner, and R. J. Wasilewski. "55-Nitinol- -The Alloy with a Memory: Its Physical Metallurgy, Properties, and Applications: A Report”. Washington: NASA, 1972.

[9] Keller et al., "A Self-Healing Poly (dimethyl siloxane) Elastomer"; Advanced Functional Materials, v. 17, p. 23992404 (2007).

[10] W. M. Kier and K. K. Smith, "Tongues, Tentacles, and Trunks: The Biomechanics of Movement in Muscular-hydrostats", Zoological Journal of the Linnean Society, Vol. 83, pp. 307-324, 1985. 
[11] Michael. O. Obaji and Shiwu Zhang. "Investigation into the Force Distribution Mechanism of a Soft Robot Gripper Modeled for Picking Complex Objects Using Shape Memory Alloy Actuators". Proceedings of the IEEE International Conference on Cybernetics, Intelligent Systems and Robotics, Automation \& Mechatronics (CIS-RAM), Manila Philippines, Nov. 11-16, 2013, P. 7. ISBN: 978-1-4799-1198-1.

[12] Tanaka, K., and Nagaki, S. (1982). "A thermo mechanical description of materials with internal variables in the process of phase Transitions", Ing.-Arch. 51 287-99.

[13] Online Journal on production of silicone elastomers. Accessed; April 2013 http://www.silicone.jp/e/catalog/pdf/rubber_e.pdf

[14] Stockwell Elastomerics: "Silicone Sponge and Silicone Rubber Gaskets, Seals, Cushions, and Material". http://www.stockwell.com/data-sheets/silicone-materials-guid e.pdf: Accessed January 1, 2014.

[15] O'Halloran A and O'Malley F (2004)., Materials and technologies for artificial muscle: a review for the mechatronic muscle project Topics in Bio-Mechanical Engineering ed P J Prendergast and P E McHugh pp 184-215.

\section{Biography}

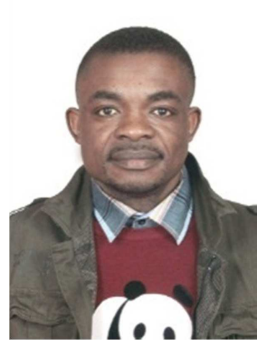

Michael. O. Obaji holds a $\mathrm{PhD}$ in Instrumentation Engineering from the University of Science \& Technology of China. He was invited in 2014 by the Ebonyi State Government of Nigeria to install and test-run four production lines of HDPE Pipes Production Machines imported from China. $\mathrm{He}$ was later invited again to be the General Manager of the facility now called Ebonyi Pipes Production Limited. He is also with the Department of Mechatronics Engineering of the Federal University, Oye-Ekiti, Nigeria. His research interests include smart materials and their applications in bio-robots; intelligent mechanics; 3D-printing \& Mechatronics systems. 